\title{
Prognostic impact of tumor-associated macrophage infiltration in non-small cell lung cancer: A systemic review and meta-analysis
}

\author{
Jiandong Mei ${ }^{1,2, *}$, Zhilan Xiao ${ }^{1,2, *}$, Chenglin $\mathrm{Guo}^{1,2}$, Qiang $\mathrm{Pu}^{1,2}$, Lin Ma ${ }^{1,2}$, Chengwu \\ Liu $^{1,2}$, Feng Lin ${ }^{1,2}$, Hu Liao ${ }^{1,2}$, Zongbing You ${ }^{3}$, Lunxu Liu ${ }^{1,2}$ \\ ${ }^{1}$ Department of Thoracic Surgery, West China Hospital, Sichuan University, Chengdu 610041, China \\ ${ }^{2}$ Western China Collaborative Innovation Center for Early Diagnosis and Multidisciplinary Therapy of Lung Cancer, Sichuan \\ University, Chengdu 610041, China \\ ${ }^{3}$ Department of Structural and Cellular Biology, Tulane University, New Orleans, LA 70112, USA \\ *These authors contributed equally to this work \\ Correspondence to: Lunxu Liu, email: Iunxu_liu@aliyun.com \\ Zongbing You, email: zyou@tulane.edu
}

Keywords: clinicopathological characteristics, non-small cell lung cancer (NSCLC), prognosis, tumor-associated macrophages (TAMs)

Received: February 24, $2016 \quad$ Accepted: April 11, $2016 \quad$ Published: April 28, 2016

\section{ABSTRACT}

Tumor-associated macrophages (TAMs) are important components of cancer microenvironment. In the present study, we searched PubMed, Embase, Cochrane library and Web of Science to perform a meta-analysis of 20 studies including a total of 2,572 non-small cell lung cancer (NSCLC) patients, in order to determine the association between TAMs and NSCLC prognosis. The combined hazard ratio (HR) of 9 studies showed that the density of total CD68+ ${ }^{+}$TAMs in the tumor islet and stroma was not associated with overall survival (OS) of the patients. However, the pooled HR of 4 studies showed that high density of $\mathrm{CD}^{+} 8^{+}$TAMs in the tumor islet predicted better OS, while the pooled HR of 6 studies showed that high density of CD68 ${ }^{+}$TAMs in the tumor stroma was associated with poor OS. A high islet/stroma ratio of CD68 ${ }^{+}$ TAMs was associated with better OS. A high density of M1 TAMs in the tumor islet was associated with better OS, while a high density of M2 TAMs in the tumor stroma predicted poor OS. These findings suggest that, although the density of total CD68 ${ }^{+}$ TAMs is not associated with OS, the localization and M1/M2 polarization of TAMs are potential prognostic predictors of NSCLC.

\section{INTRODUCTION}

Lung cancer is the most common cause of cancerrelated deaths in both men and women worldwide [1]. New therapeutic modalities, such as minimally invasive surgery and targeted therapy, have been introduced to the treatment of lung cancer during the past decades. However, the overall 5-year survival of lung cancer patients has been improved very little, especially in the advanced diseases [2]. Immunotherapy has brought up new options for lung cancer patients, including blockade of immune checkpoints like cytotoxic T-lymphocyteassociated protein 4 (CTLA-4) and programmed cell death 1 (PD-1) $[3,4]$. However, due to the complexity of the tumor microenvironment and the interactions among each tumor components, development of new combinational therapies targeting different mechanisms involved in tumor progression may open a new era of lung cancer therapy.

Tumor-associated macrophages (TAMs) are an important component of the tumor microenvironment $[5,6]$. This group of immune cells function as immune regulators in the tumor microenvironment and are potential targets of cancer immunotherapy [7]. TAMs may have both anti- and pro-tumor effects due to two distinctly different polarizations, i.e., M1 (also known as classically activated) and M2 (alternatively activated) TAMs [8]. Mills et al. recently described these two types of activated macrophages as 'Inhibit' type (M1) and 'Heal' type (M2) due to their stimulation of Th1 or Th2 type responses $[9,10]$. The M1 polarization is known to be induced by interferon- $\gamma$, lipopolysaccharide (LPS), tumor necrosis 
factor $\alpha$ (TNF- $\alpha$ ), and granulocyte-macrophage colonystimulating factor (GM-CSF) [8, 10, 11]. M1 macrophages function to promote Th1 responses with microbicidal and tumoricidal effects $[8,10,11]$. The M2 macrophages are known to be activated by interleukin-4 (IL-4), IL-10, IL-13, and prostaglandin E2 (PGE2) [11-13]. Activation of M2 macrophages induces Th2 responses and promotes tissue repair and remodeling, angiogenesis, and immune suppression, as well as tumor progression [11, 13, 14].

The dual roles of TAMs in tumor progression have been supported by both in vitro and in vivo studies using different tumor models. However, the role of TAMs in lung cancer progression remains controversial due to the discrepancies among the previous studies on TAM infiltration and lung cancer prognosis. A variety of markers (including CD68, CD163, CD204, HLA-DR, etc.) were used to identify different types of TAMs and the microdistribution (in the tumor islet, stroma, or both) of TAMs in the tumor tissues [15-19]. However, some technical pitfalls have compromised the conclusions drawn from the previous studies, such as small sample size that limited the statistical power in revealing the implications of TAMs on the clinicopathological characteristics and prognosis. Therefore, we conducted the present meta-analysis to evaluate the role of different types and distribution of TAMs in the tumor microenvironment of non-small cell lung cancer (NSCLC), through pooling data from 20 eligible studies.

\section{RESULTS}

\section{Characteristics of studies}

A total of 4,604 records were identified from different databases during primary search. These records were screened and irrelevant results were excluded as shown in the search flow diagram (Figure 1). Full-texts of 34 candidate studies were carefully reviewed and 14 of them were excluded. The remaining 20 original reports [15-19, 22-36] published between 1999 and 2014 were included in this meta-analysis. Detailed information of these studies is listed in Table 1. The mean NOS score of these included studies was 7.95.

Among the included studies, some patients were enrolled twice for different research purposes in different publications, including the studies by Dai et al. [18] and Ma et al. [27], Welsh et al. [32] and Ohri et al. [19], and Chen's two studies [33, 34]. As a result, a total of 2,572 patients were studied in all 20 included publications. Large cell lung cancer (LCLC) [36] and lung squamous cell carcinoma (SCC) [17] were the only histologic type in each of these studies, respectively. Three studies investigated lung adenocarcinoma (ADC) $[25,26,35]$. Both ADC and SCC patients were included in the remaining studies (Table 1).

CD68 was a common monocyte/macrophage marker and was used as TAM marker in 16 studies, including
4 studies of CD68 in combination with other specific macrophage markers. Double immunohistochemical staining was applied to estimate the prognostic role of different TAM polarization and survival in 3 studies, while the other studies used single immunohistochemical staining. M1 TAMs were labeled as $\mathrm{CD}^{+} 8^{+} \mathrm{HLA}-\mathrm{DR}^{+}$ cells in 2 studies and as $\mathrm{CD}^{+} 8^{+} \mathrm{iNOS}^{+}$cells in a third study. M2 TAMs were indicated as $\mathrm{CD} 68^{+} \mathrm{CD} 163^{+}$cells in 2 studies and as $\mathrm{CD} 68^{+} \mathrm{CD} 206^{+}$cells in a third study. There were 2 publications studying $\mathrm{CD} 163^{+}$and another 2 articles studying CD204+ TAMs, including one study that estimated both $\mathrm{CD} 68^{+}$TAMs and CD204+ M2 TAMs simultaneously. The role of $\mathrm{CD} 68^{+} \mathrm{TAMs}$ in both tumor islet and stroma on overall survival (OS) was studied in 9 articles. The tumor islet and stromal CD68 ${ }^{+}$TAM densities were reported in 4 and 5 articles, respectively.

\section{TAM density in the tumor islet + stroma $(\mathrm{I}+\mathrm{S})$ and survival}

Among the 20 included studies, 9 reported the relationship between $\mathrm{CD} 68^{+}$TAMs (I+S) and OS. The pooled HR of these 9 studies showed that CD68 ${ }^{+}$TAM infiltration was not associated with OS (high $\mathrm{CD}^{+} 8^{+}$ TAM density vs. low $\mathrm{CD} 68^{+}$TAM density, $\mathrm{HR}=1.32$, $95 \% \mathrm{CI}=0.89 \sim 1.97 ; P=0.17 ; \mathrm{I}^{2}=67 \%, P=0.002$; Figure 2A). The patients in 8 out of these 9 studies were grouped according to the count of $\mathrm{CD} 68^{+} \mathrm{TAMs}$ for high or low macrophage infiltration, while the study by Pei et al. [15] used tissue microarray and grouped patients as CD68 positive versus negative staining. Nevertheless, the conclusion remains unchanged after eliminating Pei's study. The pooled HR was 1.35 $\left(95 \% \mathrm{CI}=0.82 \sim 2.22, P=0.23 ; \mathrm{I}^{2}=70 \%, P=0.001\right)$ in the remaining 8 studies. There were 2 studies focused on the I+S CD $68^{+}$TAM density and DFS $[15,23]$. Another study used the protein named triggering receptor expressed on myeloid cells-1 (TREM-1) as a marker for TAMs and reported that TREM-1 was expressed only by $\mathrm{CD} 68^{+}$ TAMs in lung cancer tissue [31]. The pooled HR of these 3 studies [15, 23, 31] showed no association between the $\mathrm{I}+\mathrm{S}$ TAM density and DFS $(\mathrm{HR}=2.21,95 \% \mathrm{CI}=0.82 \sim$ 5.98, $\left.P=0.12 ; \mathrm{I}^{2}=77 \%, P=0.01\right)$.

In addition, we also assessed the association between $\mathrm{OS}$ and $\mathrm{M} 1$ or M2 polarization using the data reported in two studies $[25,27]$ that employed double immunohistochemical staining to identify M1 and M2 TAMs. Ma et al. marked M1 TAMs as CD68 ${ }^{+} \mathrm{HLA}-\mathrm{DR}^{+}$cells and M2 TAMs as $\mathrm{CD}^{2} 8^{+} \mathrm{CD} 163^{+}$cells [27]. Zhang et al. labeled $\mathrm{M} 1$ as $\mathrm{CD}^{2} 8^{+} \mathrm{iNOS}^{+}$cells and M2 TAMs as $\mathrm{CD} 68^{+} \mathrm{CD} 206^{+}$cells [25]. The pooled HR of the two studies revealed that high M1 density in lung cancer tissues predicted better OS ( $\mathrm{HR}=0.41$, $95 \% \mathrm{CI}=0.22 \sim 0.78, P=0.006 ; \mathrm{I}^{2}=27 \%, P=0.24$; Figure $2 \mathrm{~B}$ ), while the density of M2 TAM infiltration was not associated with $\mathrm{OS}$ in lung cancer patients ( $\mathrm{HR}=1.73,95 \%$ $\mathrm{CI}=0.37 \sim 7.99, P=0.48 ; P=78 \%, P=0.03$; Figure $2 \mathrm{C}$ ). 


\section{Islet TAM density and OS}

The micro-distribution of TAMs in the tumor microenvironment may play a role in lung cancer progression according to the included studies. Four publications reported the association between islet $\mathrm{CD} 68^{+}$ TAMs and OS (Table 1). The pooled HR showed that high density of $\mathrm{CD} 68^{+}$cells in the tumor islet predicted better $\mathrm{OS}\left(\mathrm{HR}=0.50,95 \% \mathrm{CI}=0.30 \sim 0.85, P=0.01 ; \mathrm{I}^{2}=83 \%\right.$, $P=0.0005$; Figure $3 \mathrm{~A}$ ). As for different TAM polarizations in tumor islet, high density of $\mathrm{CD} 68^{+} \mathrm{HLA}-\mathrm{DR}^{+} \mathrm{M} 1$ TAMs was also associated with better OS $(\mathrm{HR}=0.23$, $95 \% \mathrm{CI}=0.18 \sim 0.29, P<0.00001 ; \mathrm{I}^{2}=0 \%, P=0.49$; Figure 3B), while islet $\mathrm{CD} 68^{+} \mathrm{CD} 163^{+} \mathrm{M} 2$ density was not associated with $\mathrm{OS}(\mathrm{HR}=0.83,95 \% \mathrm{CI}=0.43 \sim 1.579$, $P=0.56 ; \mathrm{I}^{2}=71 \%, P=0.03 ;$ Figure $\left.3 \mathrm{C}\right)$.

\section{Stromal TAM density and OS}

Six studies reported stromal TAM density and OS (Table 1). The pooled HR of these studies revealed that high stromal $\mathrm{CD} 68^{+}$cell count was associated with worse OS $\left(\mathrm{HR}=1.40,95 \% \mathrm{CI}=1.08 \sim 1.82 ; P=0.01 ; \mathrm{I}^{2}=55 \%\right.$,

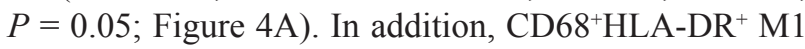
polarization was not associated with OS according to the pooled HR of 2 studies $(\mathrm{HR}=0.64,95 \% \mathrm{CI}=0.38$ $\sim 1.07, P=0.09 ; \mathrm{I}^{2}=25 \%, P=0.25$; Figure $\left.4 \mathrm{~B}\right)$. As for the study of stromal M2 density, different markers and staining techniques were applied in 6 studies. Two groups studied stromal M2 density with double staining of CD68 and CD163. The remaining 4 studies used either CD163 or CD204 as M2 markers in each of the 2 publications. The combined HR of these 6 studies showed high density of M2 TAMs in the stroma was associated with poor OS $\left(\mathrm{HR}=1.61,95 \% \mathrm{CI}=1.06 \sim 2.43, P=0.02 ; \mathrm{I}^{2}=73 \%\right.$, $P=0.003$; Figure 4C). Subgroup analysis was conducted to pool studies using different M2 markers. Only stromal CD204 ${ }^{+}$M2 TAM density was associated with OS (high density vs. low density, $\mathrm{HR}=2.01,95 \% \mathrm{CI}=1.43 \sim 2.82$, $P<0.0001 ; \mathrm{I}^{2}=0 \%, P=0.89$; Figure $4 \mathrm{C}$ ).

\section{Islet/stromal (I/S) ratio of TAM density and OS}

Four of the 20 studies also reported the association between the ratio of islet/stromal (I/S) CD68 ${ }^{+}$TAM density and OS. The pooled HR of these 4 studies showed that higher $\mathrm{I} / \mathrm{S}$ ratio of $\mathrm{CD} 68^{+} \mathrm{TAMs}$ also indicated better OS in patients with NSCLC $(\mathrm{HR}=0.28,95 \% \mathrm{CI}=0.16 \sim 0.48$, $P<0.00001 ; \mathrm{I}^{2}=55 \%, P=0.08$; Figure 5).

\section{Correlation between TAM infiltration and clinicopathological characteristics}

Some of the publications reported the association between TAM density and clinicopathological characteristics. We focused on the association between TAM density and

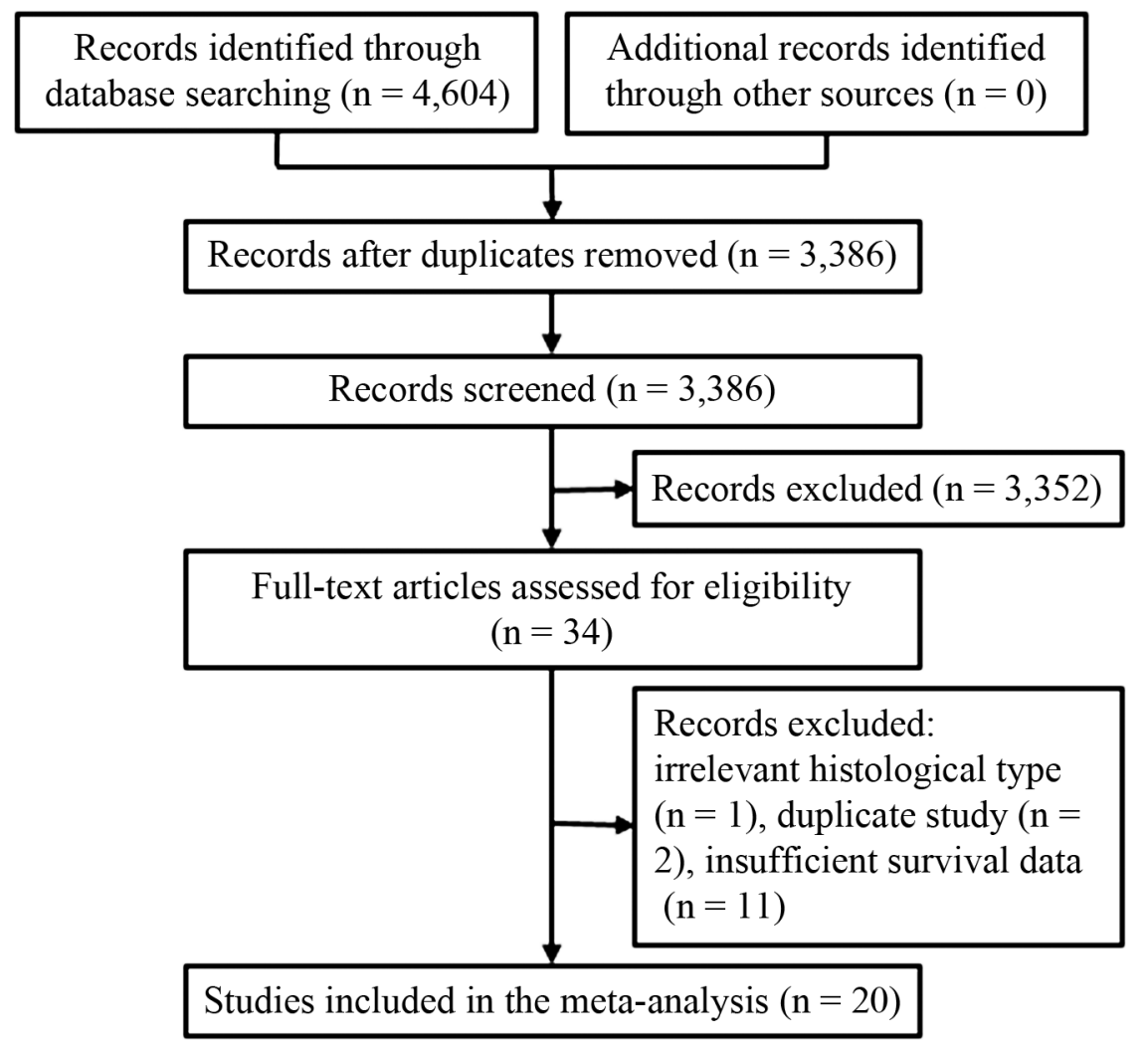

Figure 1: Flow chart for the selection of studies. A total of 20 studies were selected to determine the association between TAM density and lung cancer prognosis. 
Table 1: Characteristics of eligible studies

\begin{tabular}{|c|c|c|c|c|c|c|c|c|c|c|c|c|c|c|c|c|c|}
\hline \multirow{2}{*}{ Author } & \multirow{2}{*}{ Year } & \multirow{2}{*}{ Region } & \multirow{2}{*}{ Study Period } & \multirow{2}{*}{ Cases } & \multirow{2}{*}{ Type } & \multirow{2}{*}{ Stage } & \multirow{2}{*}{ Markers } & \multirow{2}{*}{ Group } & \multicolumn{4}{|c|}{ TAM distribution } & \multicolumn{2}{|c|}{ Polarization } & \multicolumn{2}{|c|}{ Outcome } & \multirow{2}{*}{$\begin{array}{l}\text { NOS } \\
\text { Score }\end{array}$} \\
\hline & & & & & & & & & $\mathrm{I}+\mathrm{S}$ & $\mathrm{II}$ & $\mathrm{S}$ & $\mathrm{I} / \mathrm{S}$ & M1 & M2 & DFS & OS & \\
\hline Pei $[15]$ & 2014 & China & $2003-2008$ & 417 & $\mathrm{NCLC}$ & I-III & CD68 & - vs. + & $\sqrt{ }$ & - & - & - & - & - & $\sqrt{ }$ & $\sqrt{ }$ & 9 \\
\hline Li [22] & 2014 & China & $2007.1-2008.6$ & 130 & $\mathrm{ADC}, \mathrm{SCC}$ & I-IV & CD163 & $<\mathrm{M}$ vs. $>\mathrm{M}$ & - & - & $\sqrt{ }$ & - & - & - & - & $\sqrt{ }$ & 8 \\
\hline Feng [23] & 2014 & Taiwan & $2005-2008$ & 28 & NSCLC & IIIA-N2 & CD68 & $<\mathrm{M}$ vs. $>\mathrm{M}$ & $\sqrt{1}$ & - & - & $\sqrt{ }$ & - & - & $\sqrt{ }$ & $\sqrt{ }$ & 8 \\
\hline Carus [16] & 2013 & Denmark & $2003-2006$ & 335 & NSCLC & I-IIIA & CD163 & $<\mathrm{M}$ vs. $>\mathrm{M}$ & - & 1 & $\sqrt{ }$ & - & - & - & - & $\sqrt{ }$ & 9 \\
\hline Hirayama [17] & 2012 & Japan & $2000-2006$ & 208 & SCC & I-IIIA & CD204 & $<\mathrm{M}$ vs. $>\mathrm{M}$ & - & - & $\sqrt{ }$ & - & - & - & $\sqrt{ }$ & $\sqrt{ }$ & 9 \\
\hline Paola [24] & 2012 & Brazil & N/A & 65 & NSCLC & I-IIIA & CD68 & $\begin{array}{c}\leq 4.5 \% \text { vs. }> \\
4.5 \%\end{array}$ & $\sqrt{ }$ & - & -1 & - & - & - & - & $\checkmark$ & 5 \\
\hline Zhang [25] & 2011 & China & 2003-2006 & 65 & $\mathrm{ADC}$ & I-IV & $\begin{array}{c}\text { CD68+iNOS/ } \\
\text { CD206 }\end{array}$ & $<\mathrm{M}$ vs. $>\mathrm{M}$ & $\sqrt{ }$ & - & -1 & - & $\sqrt{ }$ & $\checkmark$ & - & $\checkmark$ & 9 \\
\hline Ohtaki [26] & 2010 & Japan & $1996.1-1998.3$ & 170 & $\mathrm{ADC}$ & I-IIIA & CD68/CD204 & $<\mathrm{M}$ vs. $>\mathrm{M}$ & - & - & $\sqrt{ }$ & - & - & - & - & $\sqrt{ }$ & 8 \\
\hline $\mathrm{Ma}$ [27] & 2010 & China & 1999.6-2001.8 & 100 & NSCLC & I-IV & $\begin{array}{c}\text { CD68+HLA- } \\
\text { DR/CD163 }\end{array}$ & $<$ M vs. $>$ M & $\sqrt{ }$ & $\sqrt{ }$ & $\sqrt{ }$ & - & $\sqrt{ }$ & $\checkmark$ & - & $\sqrt{ }$ & 8 \\
\hline Dai [18] & 2010 & China & $1999.8-2001.8$ & 99 & NSCLC & I-IV & CD68 & $<$ M vs. $>$ M & $\sqrt{ }$ & $\sqrt{1}$ & $\sqrt{ }$ & $\sqrt{ }$ & - & - & - & $\sqrt{ }$ & 9 \\
\hline Ohri [19] & 2009 & UK & $\begin{array}{c}1991-1994, \\
1999 \\
\end{array}$ & 40 & NSCLC & I-IV & $\begin{array}{c}\text { CD68+HLA- } \\
\text { DR/CD163 }\end{array}$ & $<$ M vs. $>$ M & - & $\sqrt{ }$ & $\sqrt{ }$ & - & $\sqrt{ }$ & $\sqrt{ }$ & - & $\sqrt{ }$ & 8 \\
\hline Al-Shibli [28] & 2009 & Norway & 1990-2004 & 333 & NSCLC & I-IIIA & CD68 & $\begin{array}{c}<25 \% \text { vs. }> \\
25 \%\end{array}$ & - & - & 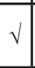 & - & - & - & - & $\checkmark$ & 8 \\
\hline Kim [29] & 2008 & Korea & $1997-1998$ & 144 & NSCLC & I-IV & CD68 & $<\mathrm{M}$ vs. $>\mathrm{M}$ & $\sqrt{ }$ & $\checkmark$ & $\sqrt{ }$ & - & - & - & - & $\sqrt{ }$ & 9 \\
\hline Kawai [30] & 2008 & Japan & 1996-2004 & 199 & NSCLC & $\mathrm{IV}^{*}$ & $\begin{array}{ll}\text { CD68 } \\
\end{array}$ & $<\mathrm{M}$ vs. $\geq \mathrm{M}$ & - & V & $\sqrt{ }$ & $\sqrt{ }$ & - & - & - & $\sqrt{ }$ & 8 \\
\hline Нo [31] & 2008 & Taiwan & $1996.9-1998.9$ & 68 & NSCLC & I-III & TREM1 & $<\mathrm{M}$ vs. $>\mathrm{M}$ & $\sqrt{ }$ & - & - & - & - & - & $\sqrt{ }$ & $\sqrt{ }$ & 7 \\
\hline Welsh [32] & 2005 & UK & $\begin{array}{c}1991-1994 \\
1999\end{array}$ & 118 & NSCLC & I-IV & CD68 & $<$ M vs. $>$ M & - & $v$ & 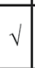 & $\sqrt{ }$ & - & - & - & $\sqrt{ }$ & 9 \\
\hline Chen [33] & 2005 & Taiwan & \begin{tabular}{|l|}
$1994.9-1996.9$ \\
\end{tabular} & 41 & $\mathrm{ADC}, \mathrm{SCC}$ & I-IV & CD68 & $<\mathrm{M}$ vs. $>\mathrm{M}$ & - & $\sqrt{1}$ & - & - & - & - & $\sqrt{ }$ & - & 7 \\
\hline Chen [34] & 2003 & Taiwan & $1994.5-1994.12$ & 35 & SCC, ADC & I-IIIA & CD68 & $<\mathrm{M}$ vs. $>\mathrm{M}$ & $\sqrt{1}$ & - & - & - & - & - & - & $\sqrt{ }$ & 7 \\
\hline Takanami [35] & 1999 & Japan & 1986-1992 & 113 & $\mathrm{ADC}$ & I-IV & CD68 & $\begin{array}{c}\leq \text { Mean vs. }> \\
\text { Mean }\end{array}$ & $\sqrt{ }$ & - & -1 & - & - & - & - & $\sqrt{ }$ & 7 \\
\hline Eerola [36] & 1999 & Finland & $1978-1995$ & 38 & LCLC & I-III & CD68 & $<\mathrm{M}$ vs. $>\mathrm{M}$ & $\sqrt{ }$ & - & - & - & - & - & - & $\sqrt{ }$ & 7 \\
\hline
\end{tabular}

Abbreviations: $\mathrm{ADC}=$ Adenocarcinoma, $\mathrm{DFS}=$ Disease free survival, $\mathrm{LCLC}=$ Large cell lung cancer, $\mathrm{I}=$ Islet, $\mathrm{M}=$ Medium, NOS $=$ Newcastle-Ottawa Scale, NSCLC $=$ Non-small cell lung cancer, $\mathrm{OS}=$ Overall survival, $\mathrm{S}=$ Stroma, $\mathrm{SCC}=$ Squamous cell carcinoma, $\mathrm{TAM}=$ Tumor-associated macrophage, $\mathrm{UK}=$ United Kingdom

A Tumor islet and stromal (I+S) $\mathrm{CDG8}^{+}$TAMs and $\mathrm{OS}$



\section{B I+S M1 TAMs and OS}

\begin{tabular}{|c|c|c|c|c|c|c|c|c|c|}
\hline Study & $\log [\mathrm{HR}]$ & SE & Weight & HR & Year & \multicolumn{4}{|c|}{$\begin{array}{c}\text { HR } \\
\text { IV, Fixed, } 95 \% \text { CI }\end{array}$} \\
\hline Ma 2010 & -1.07 & 0.36 & $80.0 \%$ & $0.34[0.17,0.69]$ & 2010 & & & & \\
\hline Zhang 2011 & -0.13 & 0.72 & $20.0 \%$ & $0.88[0.21,3.60]$ & 2011 & & & & \\
\hline Total $(95 \%$ CI) & & & $100 \%$ & $0.41[0.22,0.78]$ & & & & & \\
\hline $\begin{array}{l}\text { Heterogeneity: } \mathrm{Cl} \\
\text { Test for overall e }\end{array}$ & $\begin{array}{l}1.36, \mathrm{df}=1 \\
\mathrm{Z}=2.74(\mathrm{P}\end{array}$ & $\begin{array}{l}(\mathrm{P}=0 \\
=0.0\end{array}$ & .24); $\mathrm{I}^{2}=2$ & & & & $\begin{array}{c}0.2 \\
\text { Favours [Low density] }\end{array}$ & 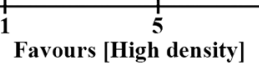 & 20 \\
\hline C I+S M2 TA & and OS & & & HP & & & & & \\
\hline Study & $\log [\mathrm{HR}]$ & $\mathbf{S E}$ & Weight & IV, Random, $95 \%$ CI & Year & & IV, Rando & $\mathrm{m}, 95 \% \mathrm{CI}$ & \\
\hline Ma 2010 & -0.13 & 0.3 & $57.2 \%$ & $0.88[0.49,1.58]$ & 2010 & & द & 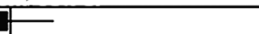 & \\
\hline Zhang 2011 & 1.45 & 0.67 & $42.8 \%$ & $4.26[1.15,15.85]$ & 2011 & & & & \\
\hline Total (95\% CI) & & & $100 \%$ & $1.73[0.37,7.99]$ & & & & & \\
\hline $\begin{array}{l}\text { Heterogeneity: Ta } \\
\text { Test for overall ef }\end{array}$ & $\begin{array}{l}0.98 ; \mathrm{Chi}^{2}= \\
\mathrm{Z}=0.70(\mathrm{P}\end{array}$ & $\begin{array}{l}4.63, \\
=0.48\end{array}$ & $\mathrm{df}=1(\mathrm{P}=$ & $.03) ; \mathrm{I}^{2}=78 \%$ & & 0.05 & $\begin{array}{c}1 \\
0.2 \\
\text { Favours [Low }\end{array}$ & 1 Favours [High density] & 20 \\
\hline
\end{tabular}

Figure 2: Forest plot of HR for TAM density in the tumor islet and stroma (I+S) and overall survival (OS). (A) The pooled HR of 9 studies showed that I+S CD68 $8^{+}$TAM density was not associated with OS (high CD68 ${ }^{+}$TAM density vs. low CD68 $8^{+}$TAM density, $\left.\mathrm{HR}=1.32,95 \% \mathrm{CI}=0.89 \sim 1.97 ; P=0.17 ; \mathrm{I}^{2}=67 \%, P=0.002\right)$. (B) High $\mathrm{I}+\mathrm{S} \mathrm{M} 1 \mathrm{TAM}$ density predicted better OS $(\mathrm{HR}=0.41$, $\left.95 \% \mathrm{CI}=0.22 \sim 0.78, P=0.006 ; \mathrm{I}^{2}=27 \%, P=0.24\right)$. C. I+S M2 TAM density was not associated with OS of lung cancer patients $(\mathrm{HR}=$ $\left.1.73,95 \% \mathrm{CI}=0.37 \sim 7.99, P=0.48 ; \mathrm{I}^{2}=78 \%, P=0.03\right)$. 
Table 2: The relationship between TAMs and clinicopathological characteristics

Patient characteristics $\quad$ Studies (Ref No.) $\quad$ Overall OR (95\% CI) $\begin{array}{ccc}\text { Heterogeneity test } \\ { } } & I^{2} \quad p \text {-value } & p \text {-value }\end{array}$

CD68 $^{+}$TAMs (I+S) and clinicopathological characteristics

\begin{tabular}{|l|c|c|c|c|c|c|}
\hline Gender (Male vs. Female) & {$[15,23]$} & $1.38(0.86,2.20)$ & 0.43 & $0 \%$ & 0.51 & 0.18 \\
\hline Age $(>60$ vs. $<60$ years) & {$[15,23]$} & $0.64(0.13,3.29)$ & 2.73 & $63 \%$ & 0.10 & 0.60 \\
\hline Histology (ADC vs. Non-ADC) & {$[15,18,23]$} & $1.47(0.64-3.39)$ & 4.83 & $59 \%$ & 0.09 & 0.37 \\
\hline p-stage (III-IV vs I-II) & {$[15,23]$} & $1.23(0.80,1.90)$ & 0.51 & $0 \%$ & 0.48 & 0.35 \\
\hline
\end{tabular}

\section{Islet $\mathrm{CD68}^{+}$TAMs and clinicopathological characteristics}

\begin{tabular}{|l|c|c|c|c|c|c|}
\hline Histology (ADC vs. Non-ADC) & {$[18,29]$} & $1.09(0.48,2.49)$ & 2.55 & $61 \%$ & 0.11 & 0.84 \\
\hline p-stage (III-IV vs I-II) & {$[18,29,32]$} & $0.52(0.34,0.81)$ & 0.41 & $0 \%$ & 0.82 & 0.004 \\
\hline
\end{tabular}

\section{Stromal CD68 $^{+}$TAMs and clinicopathological characteristics}

\begin{tabular}{|l|c|c|c|c|c|c|}
\hline Gender (Male vs. Female) & {$[26,29]$} & $2.22(1.37,3.60)$ & 0.05 & $0 \%$ & 0.82 & 0.001 \\
\hline Smoker (Yes vs. No) & {$[26,29]$} & $2.16(0.81,5.76)$ & 4.27 & $77 \%$ & 0.04 & 0.13 \\
\hline Histology (ADC vs. Non-ADC) & {$[18,29,32]$} & $1.50(0.69,3.28)$ & 7.10 & $72 \%$ & 0.03 & 0.30 \\
\hline Grade (poor vs. well) & {$[18,29,32]$} & $2.93(1.14,7.56)$ & 10.3 & $81 \%$ & 0.006 & 0.03 \\
\hline N-stage $\left(\mathrm{N}_{1-2}\right.$ vs $\left.\mathrm{N}_{0}\right)$ & {$[18,26]$} & $2.45(1.41,4.26)$ & 0.66 & $0 \%$ & 0.42 & 0.002 \\
\hline p-stage (III-IV vs I-II) & {$[18,29]$} & $1.17(0.48,2.83)$ & 2.64 & $62 \%$ & 0.10 & 0.73 \\
\hline
\end{tabular}

\section{Stromal CD204 ${ }^{+}$TAMs and clinicopathological characteristics}

\begin{tabular}{|l|c|c|c|c|c|c|}
\hline Gender $($ Male vs. Female) & {$[17,26]$} & $1.81(0.40,8.12)$ & 7.02 & $86 \%$ & 0.008 & 0.44 \\
\hline Age $(>70$ vs. $<70$ yrs) & {$[17,26]$} & $0.82(0.54,1.26)$ & 0.40 & $0 \%$ & 0.53 & 0.36 \\
\hline Smoker $($ Yes vs. No) & {$[17,26]$} & $1.50(0.09,23.81)$ & 14.0 & $93 \%$ & 0.002 & 0.77 \\
\hline T-stage $\left(\mathrm{T}_{1}\right.$ vs $\left.\mathrm{T}_{2-4}\right)$ & {$[17,26]$} & $2.83(1.52,3.72)$ & 0.15 & $0 \%$ & 0.70 & 0.0002 \\
\hline N-stage $\left(\mathrm{N}_{1-2}\right.$ vs $\left.\mathrm{N}_{0}\right)$ & {$[17,26]$} & $4.33(0.96,19.62)$ & 6.90 & $86 \%$ & 0.009 & 0.06 \\
\hline
\end{tabular}

patient demographic characteristics as well as TNM stage if the data were available for different TAM markers or micro-distribution. Both CD68 $8^{+}$TAM density and microdistribution (islet and/or stroma) in lung cancer tissues were studied. The main results of TAM infiltration and patient characteristics were summarized in Table 2. There were no association between the $\mathrm{I}+\mathrm{S} \mathrm{CD} 68^{+}$TAM density and gender (male vs. female), age ( $>60$ vs. $<60$ years old), histologic type (ADC vs. non-ADC) or TNM stage (III-IV vs. I-II). As for the islet $\mathrm{CD} 68^{+} \mathrm{TAMs}$, there was no difference between different histologic types between the low and high TAM density groups. However, there were more stage III-IV patients in the low islet $\mathrm{CD} 68^{+}$TAM group $(\mathrm{OR}=0.52,95 \%$ $\left.\mathrm{CI}=0.34 \sim 0.81, P=0.004 ; \mathrm{I}^{2}=0 \%, \mathrm{P}=0.82\right)$. In addition, high stromal CD68 ${ }^{+}$TAM density was associated with male gender $\left(\mathrm{OR}=2.22,95 \% \mathrm{CI}=1.37 \sim 3.60, P=0.001 ; \mathrm{I}^{2}=0 \%\right.$, $P=0.82)$, poor differentiation $(\mathrm{OR}=2.93,95 \% \mathrm{CI}=1.14 \sim$ $\left.7.56, P=0.03 ; \mathrm{I}^{2}=81 \%, P=0.006\right)$, and advanced lymph node stage $(\mathrm{N}$-stage; $\mathrm{OR}=2.45,95 \% \mathrm{CI}=1.41 \sim 4.26$, $P=0.002 ; \mathrm{I}^{2}=0 \%, P=0.42$ ). There were 2 studies that evaluated stromal CD204 $4^{+}$TAMs and patient characteristics. The pooled HR revealed that high stromal CD204 ${ }^{+}$TAM density was associated with advanced tumor stage (T-stage;
$\mathrm{OR}=2.83,95 \% \mathrm{CI}=1.52 \sim 3.72, P=0.0002 ; \mathrm{I}^{2}=0 \%$, $P=0.70)$.

\section{Publication bias}

Begg's funnel plot and Egger's test were employed to investigate publication bias among the included studies on $\mathrm{CD}^{+} 8^{+}$TAMs and OS. Both tests indicated no publication bias among the studies regarding the $\mathrm{I}+\mathrm{S}$ CD68 ${ }^{+}$TAMs and OS (Begg's test, $P=0.677$, Figure 6A; Egger's test, $P=0.951)$. As for the studies of the islet $\mathrm{CD}^{+} 8^{+}$TAMs and OS, Begg's test showed no publication bias $(P=0.174$, Figure 6B), whereas Egger's test revealed statistical significance $(P=0.027)$ among the studies. The studies about stromal $\mathrm{CD}^{+} 8^{+}$TAMs and OS were also evaluated with Begg's and Egger's tests and showed no evidence of publication bias (Begg's test, $P=0.348$, Figure $6 \mathrm{C}$; Egger's test, $P=0.700)$. There was no evidence of publication bias among the studies regarding the I/S ratio of $\mathrm{CD} 68^{+}$TAMs and OS (Begg's test, $P=1.000$, Figure $6 \mathrm{D}$; Egger's test, $P=0.105)$. Due to the small number of studies focused on TAM polarization or micro-distribution, the tests for publication bias were not performed. 


\section{DISCUSSION}

The treatment of lung cancer remains a thorny problem, especially for those with advanced stage diseases. Surgery is the most preferred treatment option for patients with early stage NSCLC [37, 38]. For patients with more advanced diseases, the widely accepted therapeutic strategies are chemotherapy and/or radiotherapy, while targeted therapy is an alternative for some patients with sensitive mutations [38]. Recently, immunotherapy emerged as a therapeutic promise for patients with different kinds of cancers, including NSCLC [3]. Comprehensive understanding of tumor immune microenvironment may contribute to the development of novel immunotherapeutic agents.

TAMs are important component of tumor immune microenvironment and promote tumor progression via various mechanisms, including promoting therapeutic resistance, angiogenesis, immune suppression and metastasis [7]. The multifunctional properties of TAMs in tumor progression indicate that targeting this group of immune cells may represent a novel immunotherapeutic strategy. Using a subcutaneous lung cancer model, Ren et al. found that interferon- $\gamma$ and/or celecoxib attenuated tumor growth through modulating M2/M1 TAM ratio [39]. Overexpression of Fpr2 gene favored M1 polarization and inhibited the growth of subcutaneously implanted Lewis lung cancer [40]. In addition, targeting TAMs with anti-colony-stimulating factor 1 receptor (CSF-1R) monoclonal antibody RG7155 resulted in depletion of TAMs and objective response of the tumor [41]. Based on the pre-clinical studies, multiple clinical trials are ongoing to validate the anti-tumor effects of different agents targeting macrophage recruitment, polarization, function and activation [7].

The role of TAMs varies among different solid tumors in multiple studies performed with human samples. High density of TAMs was associated with worse survival in gastric cancer and head and neck cancer, but was associated with better OS in patients with colorectal cancer [42]. Nevertheless, there are conflicting data among the studies of TAMs and their micro-distribution in lung cancer patients [15-19, 27, 32]. In order to reveal the potential role of TAMs in NSCLC prognosis, we conducted this meta-analysis to evaluate TAM infiltration and patient survival based on different markers and microdistribution in lung cancer tissues. The results showed that

\section{A Tumor islet CD68 ${ }^{+}$TAMs and OS}

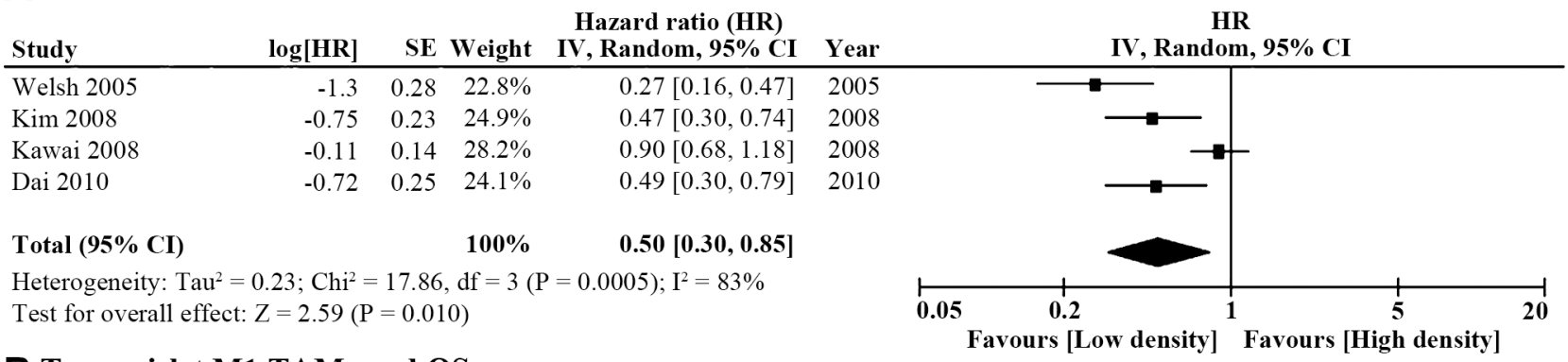

\section{$B$ Tumor islet M1 TAMs and OS}

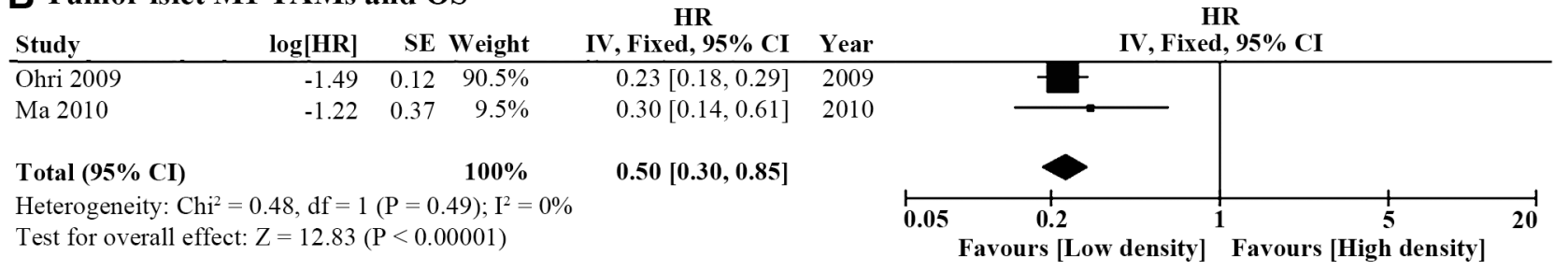

\section{Tumor islet M2 TAMs and OS}

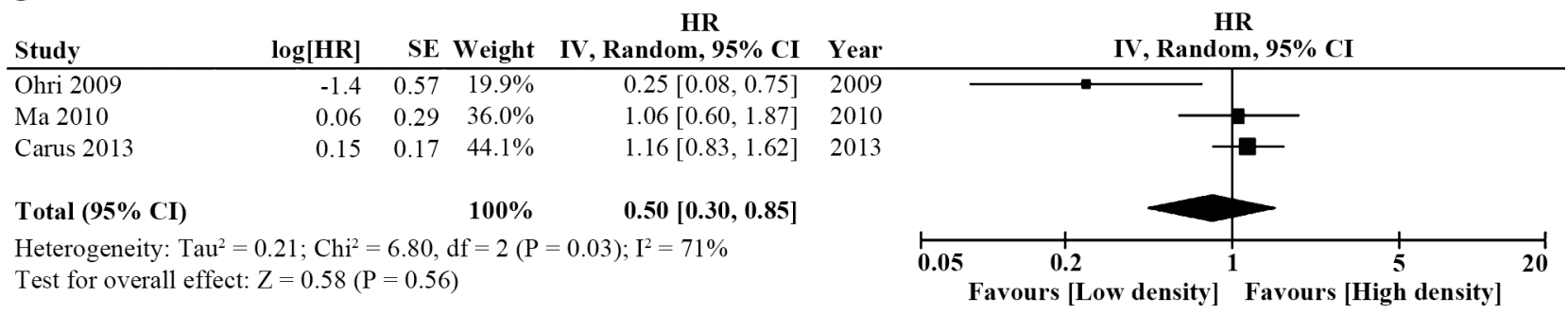

Figure 3: Forest plot of HR for TAM density in the tumor islet and OS. (A) High tumor islet CD68 $8^{+}$TAM density predicted better OS $\left(\mathrm{HR}=0.50,95 \% \mathrm{CI}=0.30 \sim 0.85, P=0.01 ; \mathrm{I}^{2}=83 \%, P=0.0005\right)$. (B) High tumor islet M1 TAM density was associated with better OS $\left(\mathrm{HR}=0.23,95 \% \mathrm{CI}=0.18 \sim 0.29, P<0.00001 ; \mathrm{I}^{2}=0 \%, P=0.49\right)$. (C) Tumor islet M2 TAM density was not associated with $\mathrm{OS}\left(\mathrm{HR}=0.83,95 \% \mathrm{CI}=0.43 \sim 1.579, P=0.56 ; \mathrm{I}^{2}=71 \%, P=0.03\right)$. 
neither I+S CD68 ${ }^{+}$TAM density nor $\mathrm{CD} 68^{+} \mathrm{CD} 163^{+} \mathrm{M} 2$ TAM density was correlated with survival. However, low

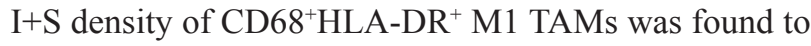
be associated with poor OS. As for the micro-distribution of TAMs and survival, low density of $\mathrm{CD}^{+} 8^{+}$TAMs and $\mathrm{CD}^{+} 8^{+} \mathrm{HLA}_{-}-\mathrm{DR}^{+} \mathrm{M} 1 \mathrm{TAMs}$ in lung cancer islet were both associated with poor OS, while islet $\mathrm{CD} 68^{+} \mathrm{CD} 163^{+}$ M2 TAM density was not correlated with prognosis. High density of stromal $\mathrm{CD}^{+} 8^{+} \mathrm{TAMs}$ or $\mathrm{CD}^{204^{+}} \mathrm{M} 2$
TAMs were associated with poor OS, whereas stromal $\mathrm{CD}_{68}{ }^{+} \mathrm{HLA}_{-D R}{ }^{+} \mathrm{M} 1$ and $\mathrm{CD}^{+} 8^{+} \mathrm{CD} 163^{+} \mathrm{M} 2 \mathrm{TAM}$ densities were irrelevant to OS. Low ratio of islet to stromal (I/S) CD68 ${ }^{+}$TAM density also predicted poor OS.

CD68 is the most commonly used marker for the study of TAMs. A total of 12 out of the 20 included studies used CD68 as macrophage marker [15, 18, 23, 24, 28-30, 32-36], while the other 4 studies used CD68 in combination with other markers for the detection of

\section{A Stromal CD68 $8^{+}$TAMs and OS}

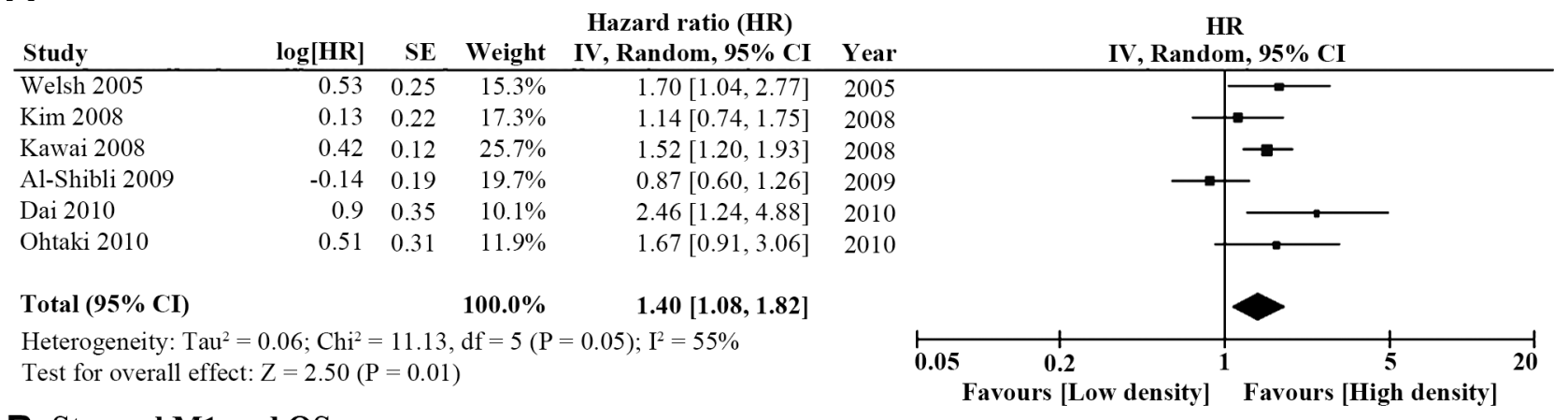

\section{B Stromal M1 and OS}

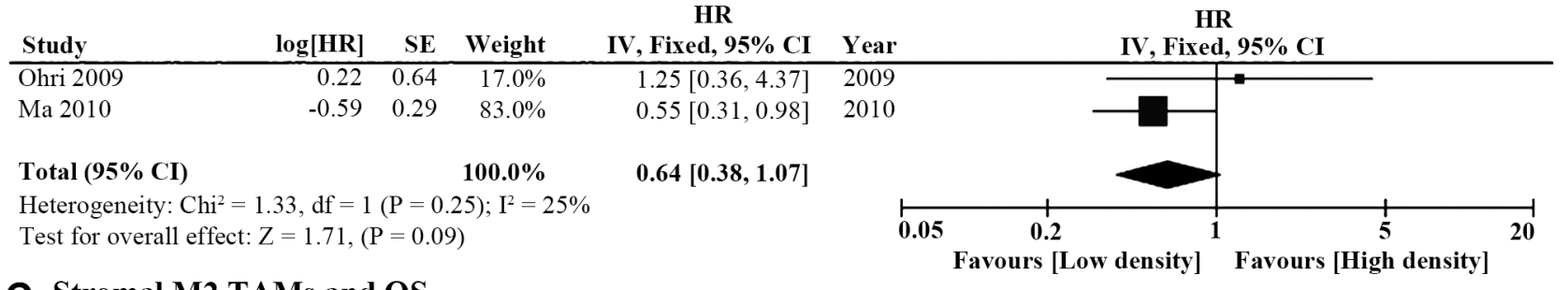

\section{Stromal M2 TAMs and OS}

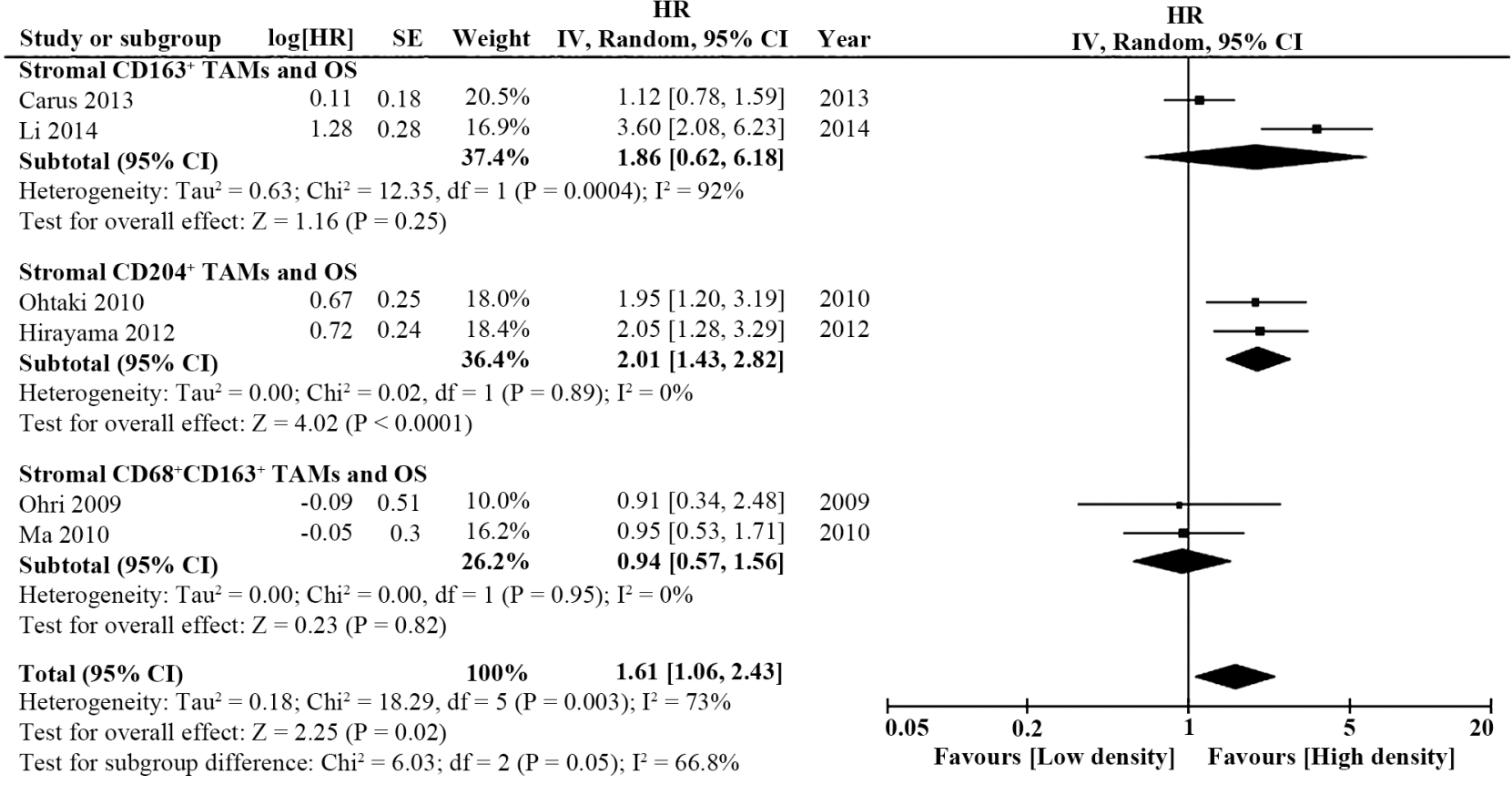

Figure 4: Forest plot of HR for TAM density in the tumor stroma and OS. (A) High stromal CD68 $8^{+}$TAM density was associated with poor OS $\left(\mathrm{HR}=1.40,95 \% \mathrm{CI}=1.08 \sim 1.82 ; P=0.01 ; \mathrm{I}^{2}=55 \%, P=0.05\right)$. (B) Stromal M1 TAM density was not associated with OS $\left(\mathrm{HR}=0.64,95 \% \mathrm{CI}=0.38 \sim 1.07, P=0.09 ; \mathrm{I}^{2}=25 \%, P=0.25\right)$. (C) High density of M2 TAMs (using different markers) in the tumor stroma was associated with poor $\mathrm{OS}\left(\mathrm{HR}=1.61,95 \% \mathrm{CI}=1.06 \sim 2.43, P=0.02 ; \mathrm{I}^{2}=73 \%, P=0.003\right)$. However, a subgroup analysis revealed that only high density of $\mathrm{CD} 204^{+} \mathrm{M} 2$ TAMs was associated with poor $\mathrm{OS}\left(\mathrm{HR}=2.01,95 \% \mathrm{CI}=1.43 \sim 2.82, P<0.0001 ; \mathrm{I}^{2}=0 \%, P=0.89\right)$. 
TAMs [19, 25-27]. Unlike some other solid tumors, the total number of $\mathrm{I}+\mathrm{S} \mathrm{CD}^{+} 8^{+}$TAMs was not associated with survival in lung cancer patients, while low islet and high stromal $\mathrm{CD}^{+} 8^{+}$TAMs were both associated with poor OS. This may be partially due to the reverse prognostic impacts of $\mathrm{CD} 68^{+}$TAMs in lung tumor islet and stroma. In addition, Gottfreid and colleagues studied the expression of CD68 in different primary cells and

Islet/stromal ratio of $\mathrm{CDC8}^{+}$TAMs and $\mathrm{OS}$

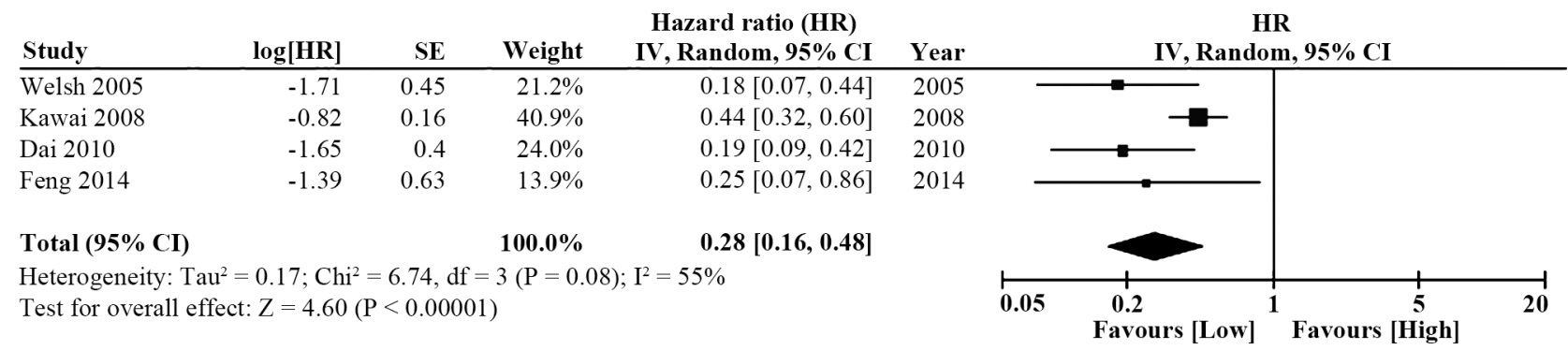

Figure 5: Forest plot of HR for the islet/stromal (I/S) ratio of CD68 ${ }^{+}$TAM density and OS. Higher I/S ratio of CD68 ${ }^{+}$cell number was associated with better $\mathrm{OS}$ in lung cancer patients $\left(\mathrm{HR}=0.28,95 \% \mathrm{CI}=0.16 \sim 0.48, P<0.00001 ; \mathrm{I}^{2}=55 \%, P=0.08\right)$.

A



C

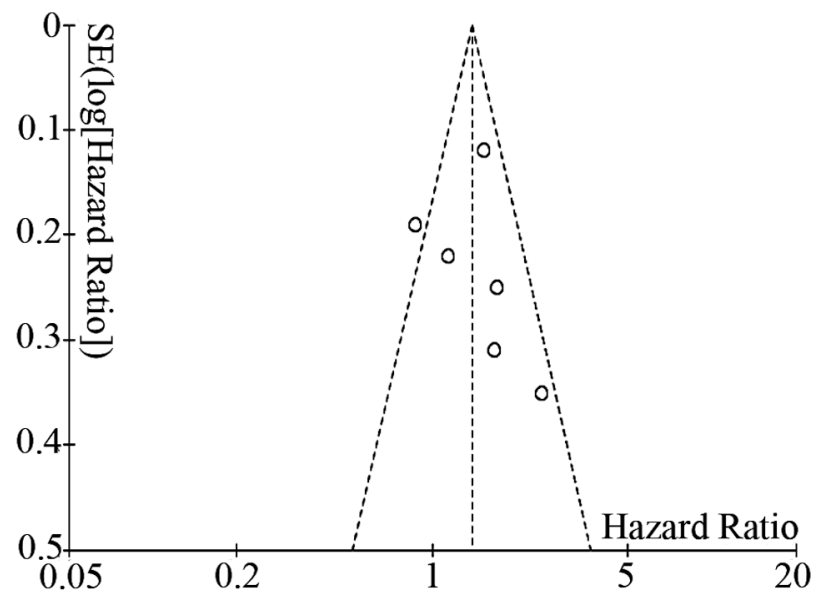

B

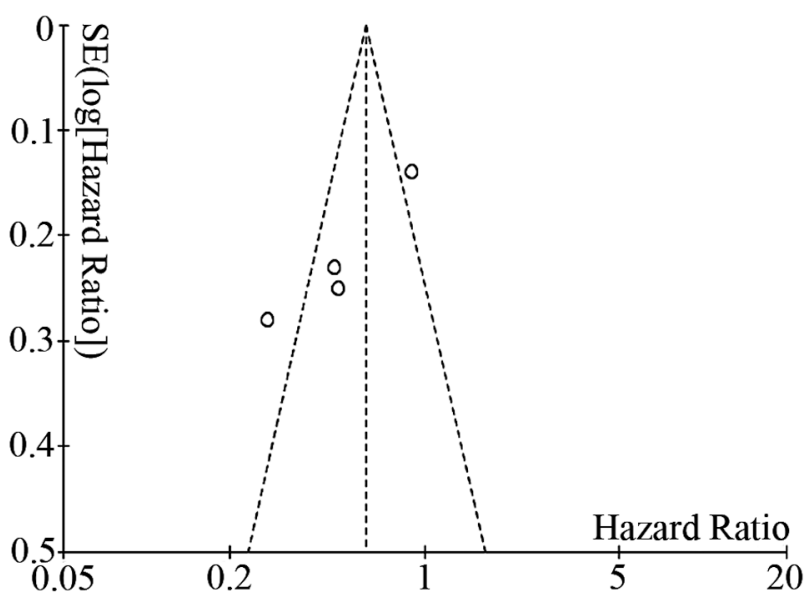

D



Figure 6: Begg's funnel plot showed no publication bias among the included studies. (A) $\mathrm{I}+\mathrm{S}$ CD68 $8^{+} \mathrm{TAMs}$ and $\mathrm{OS}$ $(P=0.677)$. (B) Islet CD68 $8^{+}$TAMs and OS $(P=0.174)$. (C) Stromal CD68 ${ }^{+}$TAMs and OS $(P=0.348)$. (D) I/S ratio of CD68 $8^{+}$TAMs and OS $(P=1.000)$. 
cancer cell lines, and found that CD68 expression was widespread, including monocytes, macrophages, fibroblasts, endothelial cells and even some cancer cells [43]. CD68 may not be a specific marker of macrophages but only enriched in this group of cells [43]. Therefore, the use of CD68 as the only macrophage marker may have some confounding effects.

In addition, several studies focused on the tumorpromoting M2 phenotype using more specific markers, including CD163 and CD204, or double staining of CD68 and CD163. Though the pooled HR of these studies confirmed the role of M2 TAMs in tumor progression, the prognostic role of different M2 markers varied according to subgroup analysis. Neither $\mathrm{CD} 68^{+} \mathrm{CD} 163^{+} \mathrm{TAMs}$ nor $\mathrm{CD} 63^{+}$TAMs were associated with patient survival. Only CD204+ ${ }^{+}$TAM density was found to be associated with OS. However, this should be further validated with larger samples.

Opposite survival effects of CD68 ${ }^{+}$TAM infiltration in the tumor islet and stroma were observed in lung cancer patients. High islet CD68 ${ }^{+}$TAM density showed similar effects as high islet M1 infiltration which was associated with better survival. However, high stromal CD68 ${ }^{+}$TAM density was related to poor OS, similar to high stromal M2 TAM infiltration. Ohri et al. demonstrated that 70\% of the TAMs in the lung cancer islet were M1 type [19]. In addition, the proportion of M2 TAMs in the lung cancer stroma was much higher than that of M1 [27]. The differences in the distribution of M1 and M2 TAMs were consistent with the survival data, reflecting their anti- and pro-tumor functions. The opposite polarization of $\mathrm{CD}^{+} 8^{+}$ TAMs in the tumor islet and stroma may also interpret the different effects of these cells on patient survival.

In these published reports, only a small number of the studies described the association between TAM infiltration and patient characteristics. There was no relationship between $\mathrm{I}+\mathrm{S} \mathrm{CD}^{+} 8^{+} \mathrm{TAM}$ density and clinicopathological features, including gender, age, histologic type and pathological stage. However, low islet $\mathrm{CD} 8^{+}$TAM density was found to be associated with more advanced pathological stage, while high stromal $\mathrm{CD} 8^{+}$TAM density was relevant to male gender, poor differentiation, and the presence of lymph node metastasis. Furthermore, high stromal CD204 $4^{+}$2 TAM density was found to be associated with more advanced T-stage. Both islet and stromal CD68 $8^{+}$TAM density and stromal CD204 M2 TAM density were associated with patient survival. Due to the limited data available, the stratified analysis to evaluate the effects of stage on TAM infiltration and patient survival was not performed.

High stromal CD68 ${ }^{+}$TAM infiltration was found to be associated with male patients. However, gender was not an independent prognostic factor according to the multivariate analysis in different studies [15, 17, 27, 29]. The exact implication of the higher stromal CD68 ${ }^{+}$TAM density in male patients remains unknown. Nevertheless, gender should be taken into consideration when selecting patients for future clinical trials.

Though we tried our best to perform a comprehensive analysis among TAM polarization, microdistribution and patient survival, there were still some shortcomings of the present study. First, the heterogeneity tests were significant in some of the pooled HRs of OS. The potential causes to explain the heterogeneity included the antibodies or methodologies used in different studies, the histologic type, origin of the patients, different edition of TNM staging system applied, and potential publication bias. Second, there was a potential risk of language bias since we only included the publications in English and Chinese. Third, some of the HRs with 95\% CIs were extracted from the Kaplan-Meier survival curve. Tumor stage is an important prognostic factor. The differences in tumor stages between the high and low TAM density groups may be a confounding factor for the observation of OS. Fourth, the number of the included studies was relatively small.

In conclusion, the present meta-analysis confirmed the potential prognostic role of TAMs in NSCLC patients. Though the total count of $\mathrm{CD}^{+} 8^{+}$TAMs was not a prognostic factor, CD68 $8^{+}$TAM density in the lung cancer islet and stroma were both associated with patient survival. Low islet and high stromal CD68 ${ }^{+}$TAM density predicted poor survival. Low islet M1 count or high stromal M2 count also showed potential association with tumor progression. Further studies with standardized methodology and larger sample size are warranted to validate the conclusions.

\section{MATERIALS AND METHODS}

\section{Literature searching strategy}

We performed electronic literature searches using PubMed, Embase, Cochrane library and Web of Science on April 16th, 2015. Studies published between January 1996 and April 2015 were selected using the following searching strategy: "lung cancer" or "lung carcinoma" or "lung neoplasm" and "Macrophage". The references of the identified articles were also reviewed to identify potentially relevant articles.

\section{Study selection}

All of the eligible articles focusing on the prognostic role of macrophages in primary NSCLC were included in this meta-analysis according to the following criteria: (1) macrophage density evaluated in primary NSCLC; (2) macrophage infiltration in NSCLC was described as high (above the cut-off value or positive) and low (below the cut-off value or negative) density; (3) overall survival (OS) and/or disease-free survival (DFS) were analyzed. The exclusion criteria were: duplicate reports, 
case reports, reviews, conference abstracts, in vitro or animal studies, non-English/non-Chinese publications, studies with duplicate cases, and studies with insufficient data for the evaluation of hazard ratio (HR) and 95\% confidence interval (CI) about OS and/or DFS. If the publications studied the same group of patients using different macrophage markers, all of them were included for marker-specific analysis.

\section{Assessment of study quality and data extraction}

Two researchers (J. Mei and Z. Xiao) independently reviewed and evaluated the included studies using the Newcastle-Ottawa Scale (NOS) [20]. Disagreement between the researchers was resolved by discussion with a third researcher. After reviewing the full text, two researchers extracted the data, including surname of the first author, publication year, origin of the study, study period, sample size, histologic type, stage, macrophage markers, grouping method, the relationship between TAM distribution and/or polarization. The HR and 95\% CI were calculated with the data extracted from survival curves, using the method described by Tierney et al. [21]. Engauge Digitizer 4.1 (http://digitizer.sourceforge.net) was used for data extraction from the survival curves.

\section{Statistical analysis}

Data analysis was carried out using Review Manager 5.3 (Cochrane Collaboration, Oxford, UK) and Stata 12.0 (Stata Corporation, Texas, US). HR with 95\% CI was used to assess the significance of TAM density on OS and DFS of the patients with NSCLC. Odds ratio (OR) was pooled by Mantel-Haenszel method to estimate the relationship between TAM density and clinicopathological characteristics. Heterogeneity of the included studies was tested by Chi-square test with $p$-value set at less than 0.10 . I-square $\left(\mathrm{I}^{2}\right)$ test was applied to assess total variation among the studies. If $p<0.10$ or $\mathrm{I}^{2}>50 \%$, the random effect model was applied to pool the data; otherwise, we chose the fixed effect model. Potential publication bias was assessed using Egger's test and Begg's test.

\section{CONFLICTS OF INTEREST}

The authors declared no conflicts of interests.

\section{GRANT SUPPORT}

L.L. is partially supported by National Natural Science Foundation of China (NSFC81172236 and NSFC81372505) and Key Science and Technology Program of Sichuan Province, China (2013SZ0005). Z.Y. was supported partially by National Institutes of Health (P20GM103518 and R01CA174714), Department of Defense (W81XWH-14-1-0050, W81XWH-14-1-0149,
W81XWH-14-1-0458 (PI: Feng Chen; Co-I: Z.Y.), and W81XWH-15-1-0444), the Developmental Fund of Tulane Cancer Center (TCC), Louisiana Cancer Research Consortium (LCRC) Fund, and Tulane's Institute of Integrated Engineering for Health and Medicine (TI2EHM). J.M. was sponsored by the China Scholarship Council (\#201406240145). Z.X. was sponsored by the China Scholarship Council (\#201406240151). The content of this article is solely the responsibility of the authors and does not necessarily represent the official views of the National Institutes of Health or the Department of Defense.

\section{REFERENCES}

1. Torre LA, Bray F, Siegel RL, Ferlay J, Lortet-Tieulent J, Jemal A. Global cancer statistics, 2012. CA Cancer J Clin. 2015; 65:87-108.

2. Leong D, Rai R, Nguyen B, Lee A, Yip D. Advances in adjuvant systemic therapy for non-small-cell lung cancer. World J Clin Oncol. 2014; 5:633-45.

3. Pardoll DM. The blockade of immune checkpoints in cancer immunotherapy. Nat Rev Cancer. 2012; 12:252-64.

4. Reck M, Paz-Ares L. Immunologic checkpoint blockade in lung cancer. Semin Oncol. 2015; 42:402-17.

5. Noy R, Pollard JW. Tumor-Associated Macrophages: From Mechanisms to Therapy. Immunity. 2014; 41:49-61.

6. Chanmee T, Ontong P, Konno K, Itano N. Tumorassociated macrophages as major players in the tumor microenvironment. Cancers (Basel). 2014; 6:1670-90.

7. Ruffell B, Coussens LM. Macrophages and Therapeutic Resistance in Cancer. Cancer Cell. 2015; 27:462-72.

8. Mantovani A, Locati M. Tumor-Associated Macrophages as a Paradigm of Macrophage Plasticity, Diversity, and Polarization. Arterioscler Thromb Vasc Biol. 2013; 33:1478-83.

9. Mills CD, Ley K. M1 and $\mathrm{m} 2$ macrophages: the chicken and the egg of immunity. J Innate Immun. 2014; 6:716-26.

10. Mills CD. M1 and M2 Macrophages: Oracles of Health and Disease. Crit Rev Immunol. 2012; 32:463-88.

11. Biswas SK, Allavena P, Mantovani A. Tumor-associated macrophages: functional diversity, clinical significance, and open questions. Semin Immunopathol. 2013; 35:585-900.

12. Liu L, Ge D, Ma L, Mei J, Liu S, Zhang Q, Ren F, Liao H, $\mathrm{Pu}$ Q, Wang T, You Z. Interleukin-17 and Prostaglandin E2 Are Involved in Formation of an M2 MacrophageDominant Microenvironment in Lung Cancer. J Thorac Oncol. 2012; 7:1091-100.

13. Sica A, Larghi P, Mancino A, Rubino L, Porta C, Totaro MG, Rimoldi M, Biswas SK, Allavena P, Mantovani A. Macrophage polarization in tumour progression. Semin Cancer Biol. 2008; 18:349-55.

14. Guo C, Buranych A, Sarkar D, Fisher PB, Wang XY. The role of tumor-associated macrophages in tumor vascularization. Vasc Cell. 2013; 5:20. 
15. Pei BX, Sun BS, Zhang ZF, Wang AL, Ren P. Interstitial tumor-associated macrophages combined with tumorderived colony-stimulating factor- 1 and interleukin- 6 , a novel prognostic biomarker in non-small cell lung cancer. J Thorac Cardiovasc Surg. 2014; 148:1208-16, e1-2.

16. Carus A, Ladekarl M, Hager H, Pilegaard H, Nielsen PS, Donskov F. Tumor-associated neutrophils and macrophages in non-small cell lung cancer: No immediate impact on patient outcome. Lung Cancer. 2013; 81:130-37.

17. Hirayama S, Ishii G, Nagai $K$, Ono S, Kojima $M$, Yamauchi C, Aokage K, Hishida T, Yoshida J, Suzuki K, Ochiai A. Prognostic impact of CD204-positive macrophages in lung squamous cell carcinoma: Possible contribution of Cd204-positive macrophages to the tumor-promoting microenvironment. J Thorac Oncol. 2012; 7:1790-97.

18. Dai F, Liu L, Che G, Yu N, Pu Q, Zhang S, Ma J, Ma L, You Z. The number and microlocalization of tumor-associated immune cells are associated with patient's survival time in non-small cell lung cancer. BMC Cancer. 2010; 10:220.

19. Ohri CM, Shikotra A, Green RH, Waller DA, Bradding P. Macrophages within NSCLC tumour islets are predominantly of a cytotoxic M1 phenotype associated with extended survival. Eur Respir J. 2009; 33:118-26.

20. Wells G, Shea B, O'Connell D, Peterson J, Welch V, Losos M, Tugwell P. The Newcastle-Ottawa Scale (NOS) for assessing the quality of nonrandomised studies in metaanalyses. Available from: http://www.ohri.ca/programs/ clinical_epidemiology/oxford.asp.

21. Tierney JF, Stewart LA, Ghersi D, Burdett S, Sydes MR. Practical methods for incorporating summary time-to-event data into meta-analysis. Trials. 2007; 8:16.

22. Li YG, Han Y, Shi Y, Fu XL, Wang JC. Prognostic significance of M2 macrophages in non-small cell lung cancer. Tumor. 2014; 34:349-56.

23. Feng PH, Yu CT, Wu CY, Lee MJ, Lee WH, Wang LS, Lin SM, $\mathrm{Fu}$ JF, Lee KY, Yen TH. Tumor-associated macrophages in stage IIIA pN2 non-small cell lung cancer after neoadjuvant chemotherapy and surgery. Am J Transl Res. 2014; 6:593-603.

24. da Costa Souza P, Parra ER, Atanazio MJ, da Silva OB, Noleto GS, Ab'saber AM, de Morais Fernezlian S, Takagaki T, Capelozzi VL. Different morphology, stage and treatment affect immune cell infiltration and long-term outcome in patients with non-small-cell lung carcinoma. Histopathology. 2012; 61:587-96.

25. Zhang BC, Yao GQ, Zhang YF, Gao J, Yang B, Rao ZG, Gao JF. M2-Polarized tumor-associated macrophages are associated with poor prognoses resulting from accelerated lymphangiogenesis in lung adenocarcinoma. Clinics. 2011; 66:1879-86.

26. Ohtaki Y, Ishii G, Nagai K, Ashimine S, Kuwata T, Hishida T, Nishimura M, Yoshida J, Takeyoshi I, Ochiai A. Stromal macrophage expressing CD204 is associated with tumor aggressiveness in lung adenocarcinoma. $\mathrm{J}$ Thorac Oncol. 2010; 5:1507-15.
27. Ma J, Liu L, Che G, Yu N, Dai F, You Z. The M1 form of tumor-associated macrophages in non-small cell lung cancer is positively associated with survival time. BMC Cancer. 2010; 10:112.

28. Al-Shibli K, Al-Saad S, Donnem T, Persson M, Bremnes RM, Busund LT. The prognostic value of intraepithelial and stromal innate immune system cells in non-small cell lung carcinoma. Histopathology. 2009; 55:301-12.

29. Kim DW, Min HS, Lee KH, Kim YJ, Oh DY, Jeon YK, Lee SH, Im SA, Chung DH, Kim YT, Kim TY, Bang YJ, Sung SW, et al. High tumour islet macrophage infiltration correlates with improved patient survival but not with EGFR mutations, gene copy number or protein expression in resected non-small cell lung cancer. Br J Cancer. 2008; 98:1118-24.

30. Kawai O, Ishii G, Kubota K, Murata Y, Naito Y, Mizuno T, Aokage K, Saijo N, Nishiwaki Y, Gemma A, Kudoh S, Ochia A. Predominant infiltration of macrophages and $\mathrm{CD} 8+\mathrm{T}$ cells in cancer nests is a significant predictor of survival in stage IV nonsmall cell lung cancer. Cancer. 2008; 113:1387-95.

31. Ho CC, Liao WY, Wang CY, Lu YH, Huang HY, Chen HY, Chan WK, Chen HW, Yang PC. TREM-1 expression in tumor-associated macrophages and clinical outcome in lung cancer. Am J Respir Crit Care Med. 2008; 177:763-70.

32. Welsh TJ, Green RH, Richardson D, Waller DA, O'Byrne KJ, Bradding P. Macrophage and mast-cell invasion of tumor cell islets confers a marked survival advantage in non-small-cell lung cancer. J Clin Oncol. 2005; 23:8959-67.

33. Chen JJW, Lin YC, Yao PL, Yuan A, Chen HY, Shun CT, Tsai MF, Chen $\mathrm{CH}$, Yang PC. Tumor-associated macrophages: The double-edged sword in cancer progression. J Clin Oncol. 2005; 23:953-64.

34. Chen JJ, Yao PL, Yuan A, Hong TM, Shun CT, Kuo ML, Lee YC, Yang PC. Up-regulation of tumor interleukin-8 expression by infiltrating macrophages: its correlation with tumor angiogenesis and patient survival in non-small cell lung cancer. Clin Cancer Res. 2003; 9:729-37.

35. Takanami I, Takeuchi K, Kodaira S. Tumor-associated macrophage infiltration in pulmonary adenocarcinoma: Association with angiogenesis and poor prognosis. Oncology. 1999; 57:138-42.

36. Eerola AK, Soini Y, Paakko P. Tumour infiltrating lymphocytes in relation to tumour angiogenesis, apoptosis and prognosis in patients with large cell lung carcinoma. Lung Cancer. 1999; 26:73-83.

37. Howington JA, Blum MG, Chang AC, Balekian AA, Murthy SC. Treatment of stage I and II non-small cell lung cancer: Diagnosis and management of lung cancer, 3rd ed: American College of Chest Physicians evidence-based clinical practice guidelines. Chest. 2013; 143:e278S-313S.

38. NCCN. NCCN Clinical Practice Guidelines in Oncology $\left(\right.$ NCCN Guidelines $^{\circledR}$ ), Non-Small Cell Lung Cancer (Version 
7.2015). [cited 2015 Aug 29]; Available from: http://www. nccn.org/professionals/physician_gls/pdf/nscl.pdf.

39. Ren F, Fan M, Mei J, Wu Y, Liu C, Pu Q, You Z, Liu L. Interferon- $\gamma$ and celecoxib inhibit lung-tumor growth through modulating M2/M1 macrophage ratio in the tumor microenvironment. Drug Des Devel Ther. 2014; 8:1527-38.

40. Liu Y, Chen K, Wang C, Gong W, Yoshimura T, Liu M, Wang JM. Cell surface receptor FPR2 promotes antitumor host defense by limiting M2 polarization of macrophages. Cancer Res. 2013; 73:550-60.

41. Ries CH, Cannarile MA, Hoves S, Benz J, Wartha K, Runza V, Rey-Giraud F, Pradel LP, Feuerhake F, Klaman I,
Jones T, Jucknischke U, Scheiblich S, et al. Targeting tumor-associated macrophages with anti-CSF-1R antibody reveals a strategy for cancer therapy. Cancer Cell. 2014; 25:846-59.

42. Zhang QW, Liu L, Gong CY, Shi HS, Zeng YH, Wang XZ, Zhao YW, Wei YQ. Prognostic significance of tumorassociated macrophages in solid tumor: a meta-analysis of the literature. PLoS One. 2012; 7:e50946.

43. Gottfried E, Kunz-Schughart LA, Weber A, Rehli M, Peuker A, Muller A, Kastenberger M, Brockhoff G, Andreesen R, Kreutz M. Expression of CD68 in nonmyeloid cell types. Scand J Immunol. 2008; 67:453-63. 